
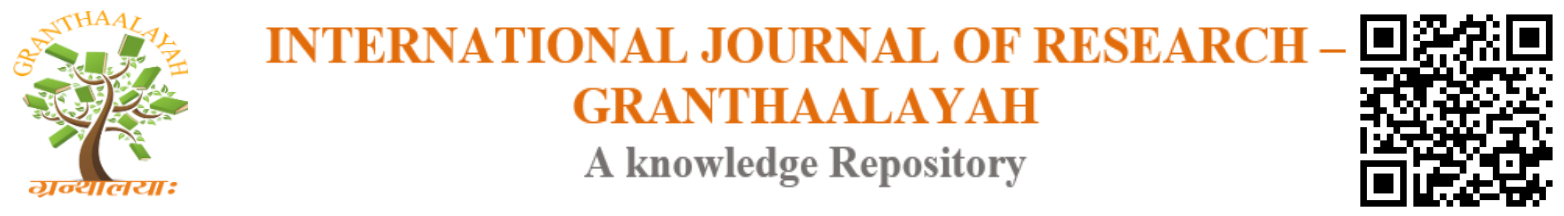

Social

\title{
PREVALENCE OF SCHISTOSOMIASIS AMONG SCHOOL CHILDREN IN BAHRI LOCALITY, SUDAN
}

\author{
Nadia A Elsammani ${ }^{1}$, Abdelghaffar Ali Adam ${ }^{2}$, Adam Abdalla Mater ${ }^{3}$, Mohamed Osman \\ Elamin 4 \\ ${ }^{1}$ PhD Student, Alzaiem Alazhari University, Sudan \\ ${ }^{2}$ Professor of Community Medicine, Shendi University, Sudan \\ ${ }^{3}$ Associate Professor, Alzaiem Alazhari University, Sudan \\ ${ }^{4}$ Faculty of Public health \&Informatics, Umm Al-Qura University
}

\begin{abstract}
Schistosomiasis is a neglected tropical disease caused by blood flukes (trematode worms) the genus Schistosoma. Schistosomiasis is a chronic, debilitating disease that affects the populations of tropical and subtropical countries, especially children at School-age. In Sudan schistosomiasis is a major health problem adversely affecting the health of vulnerable populations. A crosssectional study was conducted to determine the prevalence of schistosomiasis among school children in selected basic schools in Bahary Locality, Khartoum State, Sudan from December 2017 to January 2018. Urine and faecal samples were collected from 600 school-aged children and examined for the eggs of $S$. haematobium and $S$. mansoni using standard sedimentation and Kato technique, respectively. The overall of prevalence schistosomiasis $16,5 \%$ which the prevalence of $S$. haematobium among pupils examined was $16 \%$ and the prevalence of $S$. mansoni was $0.5 \%$. Additionally, male's reported higher prevalence $(22.7 \%)$ of $S$. haematobium than the female's $(4.1 \%)$ also males reported higher prevalence of S. mansoni female with prevalence rate $7.7 \%$ and $2.2 \%$ for males and females respectively. According to the study participant age-groups; age group 13-15 years had the highest prevalence (22\%) of S. haematobium was recorded and age group 10- 12 years comes next (18.5\%) followed by age group $6-9$ years $(7.5 \%)$; this difference of the age group as a factor was statistically significant $(\mathrm{x} 2=17.9-\mathrm{p}$ value $\mathrm{n}=0.0)$, but in $S$. mansoni age group 10-12 years had the highest $(0.8 \%)$ prevalence, also there was statistically significant $(\mathrm{P}=0.04)$. This study highlights that schistosomiasis is a public health problem among children in Bahary locality. The findings of the study suggested appropriate intervention against schistosomiasis such as mass treatment, health education and control of the intermediate snail host.
\end{abstract}

Keywords: Schistomiasis; School Children; Bahri Locality.

Cite This Article: Nadia A Elsammani, Abdelghaffar Ali Adam, Adam Abdalla Mater, and Mohamed Osman Elamin. (2019). "PREVALENCE OF SCHISTOSOMIASIS AMONG SCHOOL CHILDREN IN BAHRI LOCALITY, SUDAN.” International Journal of Research Granthaalayah, 7(9), 299-306. https://doi.org/10.29121/granthaalayah.v7.i9.2019.614. 


\section{Introduction}

Schistosomiasis is a parasitic disease, also called bilharziasis. It is caused by blood flukes (trematode flat worms) of the genus Schistosoma (s) (Chitsulo et al., 2000; Savioli et al., 2004).Six species infect humans, namely S. haematobium, S. mansoni, S. japonicum, S. intercaltum, $S$. mekongi and S. guineensis (Webster et al., 2006). The disease is transmitted from freshwater infected snails to human on contact with water. There are 2 forms of the disease in human, the urogenital and the intestinal forms, urogenital is caused by S. haematobium whilst intestinal schistosomiasis is caused by S. mansoni (Gray et al., 2011).

Schistosomiasis is considered to be second to malaria in developing countries (Sulieman et al., 2017; Hotez et al., 2010). Globally, seven hundred and seventy-nine million people are at risk of contracting the infection (Colley et al., 2014), $85 \%$ out of them in Africa. While the approximate number of disease infected people were about two hundred and seven million individual (more than $97 \%$ in Africa) in 74 countries, of them one hundred and twenty million people were diseased (Bajiro et al., 2016, Eltayeb et al.,2013). Sudan is one of Thirty-One African countries have the great burden of schistosomiasis and millions of individuals have been suffering from the disease (Bajiro et al., 2016). The highest rates of infection of schistosomiasis are often in children between the ages of 5 and 15 years (Hotez et al., 2009).

In Sudan two species of schistosomes are responsible for human schistosomiasis; S. haematobium, which cause urinary schistosomiasis, and S. mansoni, which is responsible for intestinal schistosomiasis (Deribe et al.,2011).

The objective of this study to determine the current disease prevalence among school children in and to determine the age groups most affected by the disease in Bahary locality, Khartoum, Sudan.

\section{Materials and Methods}

\subsection{Study Design}

A cross-sectional study was conducted to determine the prevalence of Schistosomiasis in selected basic schools and the most affected age groups.

\subsection{Study area}

This study was conducted in Bahary Locality (in selected basic schools) in Khartoum State, Sudan from December 2017 to January 2018 (Figures 2.1).

Bahary locality is Located between the Blue Nile in the south, River Nile State in the north. It has an area of $455 \mathrm{sq} \mathrm{km}$, and it lies between the latitudes $15^{\circ} 38^{\prime} 33^{\prime \prime} \mathrm{N}$ and longitudes $32^{\circ} 33^{\prime} 13^{\prime \prime}$. The population size is 608.817 inhabitants. The northern part of Khartoum State (Bahary) is characterized by its several and diverse economic activities. However, agriculture and industry build the backbone of the activities. For instance, Bahary is the major industrial region of Sudan, having the oil refinery and the vast lands suitable for agriculture. There are 352 schools in Bahary locality classified as public schools: 79 schools for boys and 68 for girls, and mixed 4 schools, private schools: 76 schools for boys and 66 for girls and mixed schools: 58 schools. 


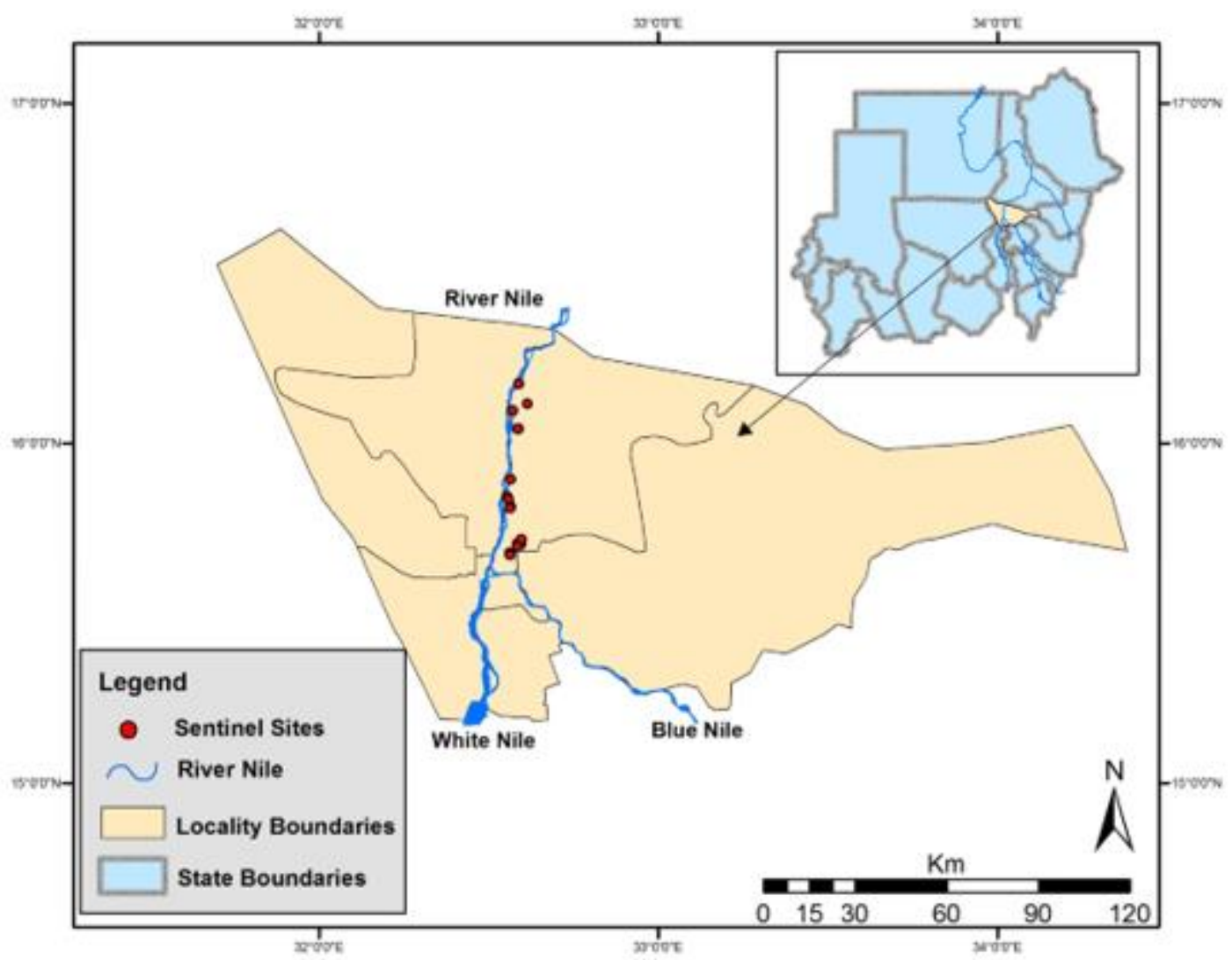

Figure (1) Map of the Study areas.

\subsection{Ethical Considerations}

The Ethical approval obtained from the Alzaiem Alazharai University, and permission obtained from the Health, Education Authorities and School leaders.

\subsection{Study Population}

The study population comprised pupils who were six to fifteen years old. The sample size was calculated for the study area using the sampling procedure recommended by WHO (Naing et al., 2006). A total of 600 schoolchildren (63.8\% males and $36.2 \%$ females) were enrolled for this study. The subjects were selected randomly from the (20) schools.

\subsection{Sample Collection}

Faecal and urine samples were collected separately in specimen bottles from school children. Each pupil was given two clean well labeled specimen containers with screw covers, for feaces and urine. The samples were collected between 10 am-12 noon in each of the schools, and transported to the laboratory for analysis. 


\subsection{Laboratory Analysis}

\subsubsection{Urine Samples Examination}

All the collected urine specimens were examined in the laboratory for the presence of Schistosoma ova using the sedimentation technique as described by Cheesbrough, (2009). The procedure involved centrifuging $10 \mathrm{ml}$ of each urine specimen at 3,000 revolutions per minute (rpm) for 5 minutes to sediment the parasite eggs. At the end of centrifugation, the supernatant was discarded and the sediment transferred onto a clean and grease-free slide, covered with a clean cover slip and viewed under a dissecting microscope, using X10 objective to focus and X100 to examine.

\subsubsection{Faecal Samples Examination}

Stool samples were examined by exploiting the modified Kato technique (Teesdale \& Amin, 1976). In such technique, only one gram of the collected faecal sample was taken and pressed through the standardized sieve with small meshes. The sieved stool sample was calibrated via small disposal syringe, which estimated to hold only 25 milligram of the sieved faecal sample. The sieved calibrated faeces then pressed out onto a clean glass-slide, and the process was repeated three times, hence, 3 slides from each sieved stool sample were prepared. Each prepared slide was then covered with another clear slide to form a "sandwich" and a gentle pressure was applied with a finger until the faecal matter spread to cover an area of $20-25 \mathrm{~mm}$ in diameter. The prepared slides were then immediately examined under a binocular microscope.

\subsection{Data Analysis}

Data obtained were analyzed using SPSS for windows version 24 and the prevalence of infection was calculated using percentages (also using the chi square test).

\section{Results}

\subsection{Sample characteristics}

A total number of 600 children aged 5-15 years consisting of $383(63.8 \%)$ males and 217(36.2\%) females. The individuals were divided into three age groups (6 -9, 10-12 and 13-15 years), representing $33.5 \%, 39.7 \%$ and $26.8 \%$ of the children, respectively.

\subsection{Prevalence of S. Mansoni and S. Haematobium, According to The Study Participants' Gender}

The overall prevalence of both forms of schistosomiasis in the study area was $16.5 \%$ from the total positives, $96(16 \%)$ and $3(0.5 \%)$ of the pupils were infected by S. haematobium and S. mansoni respectively. The distribution of the parasite for both genders showed that; out of 236 male pupils, $87(22.7 \%)$ males were infected with $S$. haematobium, while female pupils were infected with $S$. haematobium $9(4.1 \%)$ cases were recorded out of 217 screened. On other hand the prevalence of $S$. mansoni was $2(7.7 \%)$ and $1(2.2 \%)$ for males and females respectively. The prevalence of $S$. haematobium among males was significantly higher than that among females $(P<0.05)$, whereas 
There was no statistically significant difference between the prevalence of S. mansoni and the gender $(\mathrm{p}>0.05)$ (Table 1).

Table 1: The prevalence of Intestinal and urinary Schistosomiasis according to gender of pupils:

\begin{tabular}{|l|l|l|l|}
\hline \multirow{2}{*}{ Gender } & \multirow{2}{*}{ Number examined } & \multicolumn{2}{|c|}{ Number (\%) Positive for Schistosomiasis } \\
\cline { 3 - 4 } & & Intestinal (S.mansoni) & Urinary (S.haematobium \\
\hline Male & 383 & $2(0.5 \%)$ & $87(22.7 \%)$ \\
\hline Female & 217 & $1(0.5 \%)$ & $9(4.1 \%)$ \\
\hline Total & 600 & $3(0.5 \%)$ & $96(16 \%)$ \\
\hline
\end{tabular}

Chi-Square 35.533, $\mathrm{p}$ value 0.000 for Urinary (S. haematobium)

Chi-Square 0.010, p value 0.918 for Intestinal (S. mansoni)

\subsection{Prevalence of $S$. Mansoni and S. Haematobium, According to The Study Participant Age-Groups}

According to the study participant age-groups, the prevalence of $S$. mansoni only age groups 1012 years and 13-15 years were positive for the disease, and age groups $6-9$ years did not have the infection. On other hand S. haematobium infection, 37 (22\%) students were in the age group 1315 years, and the higher infestation, and the age group 10- 12 years comes next 44 (18.5\%) followed by age group $6-9$ years $15(7.5 \%)$; this difference of age group as factor was statistically significant $(\mathrm{x} 2=17.9-\mathrm{p}$ value $\mathrm{n}=0.0)$ (Table 2$)$.

Table 2: The prevalence of Intestinal and urinary Schistosomiasis according to age group of pupils:

\begin{tabular}{|l|l|l|l|}
\hline \multirow{2}{*}{ Age- group } & \multirow{2}{*}{ Number examined } & \multicolumn{2}{|c|}{ Number (\%) Positive for Schistosomiasis } \\
\cline { 3 - 4 } & & Intestinal (S.mansoni) & Urinary (S.haematobium) \\
\hline 6 -9 years & 201 & $0(0 \%)$ & $15(7.5 \%)$ \\
\hline 10- 12 years & 238 & $2(0.8 \%)$ & $44(18.5 \%)$ \\
\hline 13-15 years & 161 & $1(0.6 \%)$ & $37(22 \%)$ \\
\hline Total & 600 & $3(0.5 \%)$ & $96(16 \%)$ \\
\hline
\end{tabular}

Chi-Square $17.758, \mathrm{p}$ value 0.000 for Urinary (S.haematobium)

Chi-Square 1.612, p value 0.447 for Intestinal (S.mansoni)

\section{Discussion}

Schistosomiasis is one of the most commonly neglected tropical disease and remains a major public health problem in many developing countries, particularly among school-aged children in sub-Saharan Africa with serious impact on their nutritional status academic performance, and normal growth (WHO,2015). In this cross-sectional survey, the most important results obtained was that the prevalence of Schistosomiasis is S. haematobium. The prevalence of S. haematobium and $S$. mansoni was being $16 \%$ and $0.5 \%$ respectively. The findings were similar to the study conducted in the Um-Asher area the overall prevalence of $S$. heamatobium (12.9\%) while for $S$. mansoni was (2.9\%) (Hamza et al.,2018).

A lower prevalence rate of $S$. mansoni in this study showed compared to the research findings reported in other areas of Sudan , 51.5\% among school children in Southern Sudan, in Lui (West Equatoria region), while the prevalence of $S$. haematobium was $0.0 \%$ (no cases of $S$. hematobium 
infection was detected ) (Deganello et al.,2007). New Halfa agricultural scheme, no infection with S. heamatobium and there was a high infection with S. mansoni (27.4\%) (Afifi et al., 2016). In this study the variations in the prevalence may be due to some factors such as: difference in water contact habit, toilet utilization and ecological distribution of snails in the study area (low land and hot) this made low availability of vector snails; however the snail (Biomphalaria pfeifferi) responsible for the transmission of $S$. mansoni infection is more prevalent in areas $2000 \mathrm{~m}$ above sea level and the optimal temperature for its development and survival is around $25^{\circ} \mathrm{C}$. Above $30^{\circ} \mathrm{C}$ snail mortality increases (Brooker,2007).

The present study shows that it was gender and age dependent. The prevalence of infection for males was higher than females (Amuta and Houmsou, 2014; John et al., 2008; Raso et al., 2005, Nyati-Jokomoa and Chimbarib, 2017). This result could be due to the fact that males make more frequent contact with water than females because in traditional Sudan settings, females are mostly associated with indoor activities than males. The males are fond of going into the streams and ponds to swim, bath, fishing, and irrigation, thereby becoming more exposed to the snail intermediate hosts (cercaria) in such water bodies.

The age group 13-15 was found to be higher student's infection with $S$. hematobium, while the age-group with the highest prevalence of S. mansoni was 10-12 years, this shows that the prevalence of infection increased from younger to older pupils. In other studies reported high prevalence Schistosomiasis among age groups (9-12) years (Tamomh et al., 2017; Ismaila and Danlami, 2015), anther studies reported the high prevalence among the age group was 10-14years (Nmorsi et al.,2005; Sarkinfada et al.,2009). In addition, the study found a significant difference between three age groups (6-9, 10-12, and 13-15 years). The finding similar result was reported by Senghor et al (Senghor et al., 2014).

\section{Conclusion}

The present study shows that children at both genders are at risk of schistosomiasis transmission, because schistosomiasis is still prevalent in the study area among school those children. $22.7 \%$ males were found to be positive for $S$. haematobium, while $4.1 \%$ out of 217 female pupils screened were infected with $S$. haematobium. On other hand the prevalence of $S$. mansoni was $7.7 \%$ and $2.2 \%$ for males and females respectively. The highest prevalence was noted from 13-15 years age group, of the children and the male pupils was reported higher prevalence than the female pupils in the study area.

This study shows the schistosomiasis Control Program of the Bahary locality need an integrated control program to reduce the prevalence. Such a program should include health education program and mass treatment for all basic schools in the study area, in addition to that will be applied methods to control the intermediate snail host.

\section{Acknowledgements}

We would like to thank Alzaiem Alazhari University and local authority (Health, Education and School leaders). We also would like to acknowledge the staff and students (directors and study participants), and we are grateful for their cooperation. 


\section{References}

[1] Afifi. A, Ahmed.A, Sliman.Y, and Pengsakul.T, (2016). Epidemiology of Schistosomiasis among Villagers of the New Halfa Agricultural Scheme, Sudan. Iran J Parasitol (11). 110-115.

[2] Amuta, E.U., and Houmsou, R.S. (2014). Prevalence, intensity of infection and risk factors of urinary schistosomiasis in preschool and school aged children in Guma Local Government Area, Nigeria. Asian Pac. J. Trop. Med. 7 (1), 34-39.

[3] Bajiro, M, Dana. D, Ayana. M, Emana. B, Mekonnen. Z, Zawdie. B, Garbi. A, Kure. A, Zeynudin. A. (2016). Prevalence of Schistosoma mansoni infection and the therapeutic efficacy of praziquantel among school children in Manna District, Jimma Zone, southwest Ethiopia. Parasites $\&$ vectors, $9(1)$ : 560.

[4] Brooker, S. (2007). Spatial epidemiology of human schistosomiasis in Africa: Risk models, transmission dynamics and control. Transactions of the Royal Society of Tropical Medicine and Hygiene, 101, 1-8. doi: 10.1016/j.trstmh.2006.08.004

[5] Cheesbrough, M. (2009). District laboratory practice in tropical countries. Part2. Cambridge University Press p.357.

[6] Chitsulo L, Engels D, Montressor A, Savioli L. (2000). The global status of schistosomiasis and its control. Acta Trop.77(1): 41-51.

[7] Colley DG, Bustinduy AL, Secor WE, King CH. (2014). Human schistosomiasis. Lancet 383: 2253-2264.

[8] Deganello. R, Crucian. M, Beltramell. C, Duncan. O, Oyugi. V, Oyugi and Montresor. A. (2007), Schistosoma hematobium and S. mansoni among Children, Southern Sudan. Emerging Infectious Diseases, (13), 1504 - 1506.

[9] Deribe K, Eldaw A, Hadziabduli S, Kailie E, Omer MD, Mohammed AE. (2014). High prevalence of urinary schistosomiasis in two communities in South Darfur: implication for interventions. Parasites \& Vectors. (1): 14.doi: 10.1186/1756-3305-4-14.

[10] Eltayeb, N.M., Mukhtar M.M., Mohamed A.B. (2013). Epidemiology of schistosomiasis in Gezira area Central Sudan and analysis of cytokine profiles. Asian Pacific journal of tropical medicine, 6(2): .119-125.

[11] Hamza A. E, Elfadel. E, Nahied.E, and Dahab.R.. (2018). Prevalence of Schistosomiasis and the Associated Risk Factors among School Children in Um-Asher area, Khartoum, Sudan. International Journal of Advances in Science Engineering and Technology 6, 2321 -8991.

[12] Hotez PJ, Alvarado M, Basanez MG, Bolliger I, Bourne R, Bousssinesq M. (2014). The global burden of disease study 2010: Interpretation and implications for the neglected tropical diseases. PLoS Negl Trop DIS.;8: e2865.

[13] Hotez PJ, Kamath A. (2009). Neglected tropical diseases in sub-Saharan Africa: Review of their prevalence, distribution, and disease burden. PLoS Negl Trop Dis. 3(8): e412.

[14] Ismaila. Y, and Danlami .M, (2015). Prevalence of Schistosomiasis in primary school pupils in riverine areas of Sokoto State. Nigeria. World Academic Journal of Community Health and Epidemiology,1(2), 197-201.

[15] John. R, Ezekiel. M, Philbert. C, P., Andrew, A. (2008). Schistosomiasis transmission at high altitude crater lakes in Uganda Western Uganda. BMC Infect. Dis. 8, 110.

[16] Naing L, Winn T, Rusli BN. (2006). Practical Issues in Calculating the Sample Size Prevalence Studies. Archives of Orofacial Sciences; 1: 9-14.

[17] Nmorsi OPG, Egwunyenga OA, Ukwandu NCD, Nwokolo NQ.Urinary.(2005). Urinary schistosomiasis in a rural community in Edo state, Nigeria. Eosinophiluria as a diagnostic marker. African J. Biotech,4:183-1186.

[18] Nyati-Jokomoa. Z, and Chimbarib.M. J,. (2017).Risk factors for schistosomiasis transmission among school children in Gwanda district, Zimbabwe. Acta Tropica .175 .84-90.

[19] Raso, G., Utzinger, J., Silué, K., Ouattara, M., Yapi, M., Toty, A., Matthys, B., Vounatsou,P., Tanner, M., N'Goran, E.K.(2005).Disparities in parasitic infections, perceived ill health and access 
to health care among poorer and less poor schoolchildren of rural Cô te d'Ivoire. Trop. Med. Int. Health 10, 42-57.

[20] Sarkinfada F, Oyebanji A, Sadiq IA, Ilayasu Z. (2009). Urinary Schistosomiasis in the Danjarima Community in Kano Nigeria. J. Infect dev. Series:3(6):452-457

[21] Savioli. L, Albonico.M, Engels.D, and Montresor. A. (2004). "Progress in the prevention and control of schistosomiasis and soil-transmitted helminthiasis," Parasitol. Int., vol. 53, pp. 103-113.

[22] Senghor B, Diallo A, Sylla SN, Doucouré S, Ndiath MO, Gaayeb L,. (2014). Prevalence and intensity of urinary schistosomiasis among school children in the district of Niakhar, region of Fatick, Senegal. Parasit Vectors.; 7:5.

[23] Sulieman Y, Eltayeb RE, Pengsakul T, Afif A, Zakaria MA, Khairala MA. (2014). Schistosomiasis as a disease and its prevalence in Sudan: An overview. J of Coastal Life Medicine.5(3):129-133.

[24] Tamomh. A.G, Yousfi .S.R, Abakar .A.D, and Nour.B.Y.(2017).Prevalence of Intestinal Schistosomiasis among Basic School Children in White Nile Sugar Scheme a New Irrigation Project, White Nile State, Sudan. Biology and Medicine 10:1.

[25] Teesdate, C.H. and Amin, M.A. (1976). Comparison of the Bell technique, a modified Kato thick smear and digestion method for the field diagnosis of Schistosoma mansoni. Journal of Helminthology, 50, 17-20.

[26] Webster BL, Southgate VR, Littlewood DT. (2006). A revision of the interrelationships of Schistosoma including the recently described Schistosoma guineensis. Int J Parasitol.36:947-955

[27] World Health Organization. (2015). Investing to overcome the global impact of neglected tropical diseases: third WHO report on neglected tropical diseases. Genava: World Health Organization. [Online] Available from: http://who.int/neglected_diseases/9789241564861/en/. 